\title{
Outcomes after totally minimally invasive versus hybrid and open Ivor Lewis oesophagectomy: results from the International Esodata Study Group
}

\author{
Berend J. van der Wilk (D) ${ }^{1, *}$, Eliza R. C. Hagens (D) ${ }^{2}$, Ben M. Eyck (D) ${ }^{1}$, Suzanne S. Gisbertz ${ }^{2}$, Richard van Hillegersberg (D) ${ }^{3}$, \\ Philippe Nafteux ${ }^{4}$, Wolfgang Schröder ${ }^{5}$, Magnus Nilsson (iD ${ }^{6,7}$, Bas P. L. Wijnhoven ${ }^{1}$, Sjoerd M. Lagarde ${ }^{1, *}$ and \\ Mark I. van Berge Henegouwen ${ }^{2}$; on behalf of the International Esodata Study Group Collaborators

\begin{abstract}
${ }^{1}$ Department of Surgical Oncology and Gastrointestinal Surgery, Erasmus MC Cancer Institute, Rotterdam, the Netherlands
${ }^{2}$ Department of Surgery, Amsterdam University Medical Centre, University of Amsterdam, Amsterdam Cancer Centre, Amsterdam, the Netherlands

${ }^{3}$ Department of Surgery, University Medical Centre Utrecht, Utrecht, the Netherlands

${ }^{4}$ Department of Thoracic Surgery, University Hospitals Leuven, Leuven Cancer Institute, Leuven, Belgium

${ }^{5}$ Department of General, Visceral, Cancer and Transplantation Surgery, Faculty of Medicine and University Hospital Cologne, University of Cologne, Cologne, Germany

${ }^{6}$ Department of Clinical Science, Intervention and Technology, Karolinska Institutet, Karolinska University Hospital, Stockholm, Sweden

${ }^{7}$ Department of Upper Abdominal Diseases, Karolinska University Hospital, Stockholm, Sweden
\end{abstract}

*Correspondence to: Department of Surgery, Erasmus MC-University Medical Centre, Dr Molewaterplein 40, 3015GD, PO Box 2040, Suite Na-2119, Rotterdam, the Netherlands (e-mail: b.vanderwilk@erasmusmc.nl and s.lagarde@erasmusmc.nl)

The International Esodata Study Group Collaborators are co-authors of this study and are listed under the heading Collaborators.

\begin{abstract}
Background: Large studies comparing totally minimally invasive oesophagectomy (TMIE) with laparoscopically assisted (hybrid) oesophagectomy are lacking. Although randomized trials have compared TMIE invasive with open oesophagectomy, daily clinical practice does not always resemble the results reported in such trials. The aim of the present study was to compare complications after totally minimally invasive, hybrid and open Ivor Lewis oesophagectomy in patients with oesophageal cancer.

Methods: The study was performed using data from the International Esodata Study Group registered between February 2015 and December 2019. The primary outcome was pneumonia, and secondary outcomes included the incidence and severity of anastomotic leakage, (major) complications, duration of hospital stay, escalation of care, and 90-day mortality. Data were analysed using multivariable multilevel models.

Results: Some 8640 patients were included between 2015 and 2019. Patients undergoing TMIE had a lower incidence of pneumonia than those having hybrid (10.9 versus 16.3 per cent; odds ratio (OR) 0.56, 95 per cent c.i. 0.40 to 0.80 ) or open (10.9 versus 17.4 per cent; OR $0.60,0.42$ to 0.84 ) oesophagectomy, and had a shorter hospital stay (median 10 (i.q.r. 8-16) days versus $14(11-19)$ days $(P=0.041)$ and $11(9-16)$ days $(P=0.027)$ respectively). The rate of anastomotic leakage was higher after TMIE than hybrid (15.1 versus 10.7 per cent; OR $1.47,1.01$ to 2.13 ) or open (15.1 versus 7.3 per cent; OR 1.73, 1.26 to 2.38 ) procedures.

Conclusion: Compared with hybrid and open Ivor Lewis oesophagectomy, TMIE resulted in a lower pneumonia rate, a shorter duration of hospital stay, but higher anastomotic leakage rates. Therefore, no clear advantage was seen for either TMIE, hybrid or open Ivor Lewis oesophagectomy when performed in daily clinical practice.
\end{abstract}

\section{Introduction}

Neoadjuvant therapy plus oesophagectomy is the cornerstone of potentially curative treatment for patients with oesophageal cancer $^{1-4}$. An oesophagectomy, however, is associated with substantial morbidity, mortality, and lasting symptoms, with reduced health-related quality of life $\mathrm{e}^{5-7}$. Furthermore, it is known that postoperative complications might have detrimental prognostic consequences ${ }^{8-11}$. To reduce the risk of postoperative complications, a variety of minimally invasive surgical techniques have evolved over time ${ }^{12}$. Totally minimally invasive oesophagectomy (TMIE) resulted in short-term benefits (such as fewer in-hospital pulmonary infections, less pain, and less intraoperative blood loss) compared with open oesophagectomy in two RCTs using the McKeown technique ${ }^{13,14}$. In a population-based setting, TMIE was associated with an increase in rates of reoperation, major complications, and pulmonary complications ${ }^{15,16}$. A recent report ${ }^{17}$ has shown show that an intrathoracic anastomosis performed using minimally invasive techniques has a long proficiency gain curve and high leak rates during the learning curve phases. This may explain why daily clinical practice does not resemble the complication rate reported in the randomized setting. 
A hybrid minimally invasive approach, in which an open thoracic phase (thoracotomy) is combined with a minimally invasive abdominal phase (laparoscopy), was compared with open oesophagectomy in the MIRO trial ${ }^{18}$. Use of hybrid oesophagectomy resulted in a decrease in major complications, specifically major pulmonary complications. Thus, both McKeown TMIE and Ivor Lewis hybrid oesophagectomy seem to have advantages over open oesophagectomy, particularly related to the incidence of pneumonia and/or pulmonary complications ${ }^{13,14,18}$. In the hybrid Ivor Lewis approach, the intrathoracic anastomosis is performed via a thoracotomy, and as such it can be hypothesized that there may be a lower anastomotic leakage rate than is associated with totally minimally invasive Ivor Lewis oesophagectomy. However, the thoracotomy could result in more pulmonary complications (such as pneumonia) than occur after thoracoscopy.

The International Esodata Study Group (IESG), which consists of high-volume oesophagectomy centres, previously reached consensus on definitions of complications after oesophagectomy ${ }^{19,20}$. All participating centres now register complications after oesophagectomy according to the definitions and standards of the IESG. The primary aim of the present study was to compare the incidence of postoperative pneumonia between TMIE, hybrid, and open Ivor Lewis oesophagectomy using data from the IESG. An additional aim was to assess and compare the rate and severity of anastomotic leakage, the rate of (major) complications, duration of hospital stay, rate of escalation of care, readmission rate within 30 days, and 90-day mortality.

\section{Methods}

\section{Esophagectomy Complications Consensus Group database}

This international cohort study was undertaken using data from the Esophagectomy Complications Consensus Group (ECCG) database. This database was developed by all contributing centres who are part of the IESG. Outcomes were reported according to the STROBE reporting guidelines for reporting observational research ${ }^{21}$. The IESG currently consists of 61 highvolume centres from 21 countries. All centres had previously signed an agreement to meet all requirements of the institutional ethics committee to supply anonymized patient information to the database. The publications and audit subcommittee of the International Society for Diseases of the Esophagus research and database committee approved the present study and supplied all of the original data required for it.

Complications after oesophagectomy were reported in a standardized manner and uniformly through web-based data retrieval forms. Complications were registered according to the uniform definitions of the ECCG ${ }^{19}$. All complications occurring within 30 days of surgery or during postoperative hospital stay were reported.

\section{Patients}

Patients registered between February 2015 and December 2019 were included in this study. Only those who underwent potentially curative oesophagectomy using the Ivor Lewis approach (abdominal, open/laparoscopic approach and right-sided thoracotomy/thoracoscopy with intrathoracic anastomosis) were included. Patients who had palliative or transhiatal oesophagectomy, those who underwent definitive chemo(radio)therapy, patients who underwent hybrid oesophagectomy consisting of laparotomy and thoracoscopy, those who had an oesophageal conduit other than stomach, and patients who had a neck anastomosis were excluded from further analysis. Three separate groups were defined based on the procedure: TMIE via laparoscopy and thoracoscopy; laparoscopically assisted oesophagectomy via laparoscopy and thoracotomy (hybrid); and open oesophagectomy via laparotomy and thoracotomy.

\section{Outcomes}

The primary outcome of the present study was pneumonia, defined as new 'lung infiltrates plus clinical evidence that the infiltrate is of an infectious origin, which include the new onset of fever, purulent sputum, leucocytosis, and decline in oxygenation', according to the definition of the American Thoracic Society, the Infectious Diseases Society of America, and as used uniformly by the IESG in previous ECCG publications ${ }^{19,22,23}$.

Secondary outcomes were the rate and severity of anastomotic leakage, rate of complications and rate of major complications, duration of hospital stay, rate of escalation of care (transfer of patient to higher level of care, such as from ward to ICU), readmission rate within 30 days after hospital discharge, and 90-day mortality. Finally, an overview was undertaken of all postoperative complications that are uniformly registered in the ECCG database.

Anastomotic leakage was defined as 'a full thickness gastrointestinal defect involving the esophagus, anastomosis, staple line or conduit irrespective of presentation or method of identification', according to the ECCG definitions ${ }^{19}$. The severity of anastomotic leakage was categorized into three types ${ }^{19}$ : type I, a local defect without needing to change treatment, or treated medically or with dietary modification; type II, a local defect requiring nonsurgical intervention (such as percutaneous drainage, stent placement or packing of incision); and type III, a local defect requiring surgical therapy. Major complications were defined as any postoperative complication graded at least IIIb according to the Clavien-Dindo classification, requiring an intervention under general anaesthesia ${ }^{24}$.

\section{Statistical analysis}

Continuous variables are presented as median (i.q.r.) and categorical data as frequencies with percentages. To assess the influence of surgical approach on postoperative complications, logistic multilevel model analysis was used for categorical outcomes and linear multilevel model analysis for linear outcomes. A hospital-specific random intercept and a hospital-specific random slope for surgical approach were used in the models to adjust for interhospital variability for all outcomes. Furthermore, adjustments were made for fixed effects, including sex, age, WHO performance status, clinical T category, clinical N category, tumour location, preoperative treatment, and tumour histology. Associations are presented as adjusted odds ratios for categorical outcomes, and standardized coefficients for linear outcomes, all with corresponding 95 per cent confidence intervals.

The variance of the multilevel models, which encompasses how much of the variance in outcomes can be explained by the variability of interhospital differences, was assessed. The variance in a model measures the average spread of each value from the mean. According to the latent variable method, the variance is divided by the variance plus a constant quantity $\left(\pi^{2} / 3=3.29\right)$. Therefore, a variance of, for example, 0.32 means that 8.9 per cent $(0.32 /(0.32+3.29))$ of the variation in the outcome is attributable to differences between hospitals ${ }^{25}$. The larger this value, the more the variance can be explained by interhospital differences. The outcomes were adjusted for such interhospital differences in the multilevel models. Incomplete cases are efficiently handled 
by multilevel model analysis ${ }^{26}$. All tests were two-sided and $\mathrm{P}<0.050$ was considered statistically significant. All statistical analyses were undertaken in $\mathrm{R}$ version 3.6.2 ( $\mathrm{R}$ Core Team, $\mathrm{R}$ Foundation for Statistical Computing, Boston, MA, USA) using the lme4 package.

\section{Results}

During the study interval, the IESG registered 8640 patients who underwent oesophagectomy in 39 hospitals from 20 countries. Of these, 4733 patients fulfilled the study requirements: 1472 patients underwent TMIE, 1364 hybrid oesophagectomy, and 1897 open Ivor Lewis oesophagectomy (Fig. 1). Baseline characteristics of the included patients are shown in Table 1.

\section{Interhospital variability}

Participating centres carried out a median of 181 (i.q.r. 120-325) procedures during the study interval. TMIE was performed in 31 hospitals, hybrid oesophagectomy in 31, and open oesophagectomy in 36 hospitals. The number of TMIE procedures undertaken during the study interval ranged from 3 to 367, with median of 46 (30-129) per hospital; 25 hospitals undertook 20 or more TMIE procedures. For hybrid oesophagectomy, the number of procedures ranged from 1 to 599, with a median of 16 (3-49) per hospital; 15 hospitals performed at least 20 hybrid procedures. Figure S1 summarizes the variance for all outcomes, explaining how much of the variability in results after each surgical approach is explained by interhospital variability. The rates of postoperative complications for each group are summarized in Table 2, and relevant comparisons are shown in Table 3. Table S4 summarizes the univariable analyses of all comparisons.

\section{TMIE versus hybrid Ivor Lewis oesophagectomy}

The pneumonia rate was lower among patients who had TMIE, and this group had a shorter hospital stay than those who underwent hybrid oesophagectomy (Tables 2 and 3). The anastomotic leakage rate was higher for TMIE than hybrid oesophagectomy. The severity of anastomotic leakage, rate of any complications, rate of major complications, rate of escalation of care, readmission rate within 30 days, and 90-day mortality rate were comparable in these two groups.

\section{TMIE versus open Ivor Lewis oesophagectomy}

The pneumonia rate was lower for patients who underwent TMIE and the hospital stay shorter than for those who had open oesophagectomy. The anastomotic leakage rate was higher for patients undergoing TMIE. Other outcomes were comparable between the two groups (Tables 2 and 3).

\section{Hybrid versus open Ivor Lewis oesophagectomy}

The rates of pneumonia and anastomotic leakage were similar for hybrid and open Ivor Lewis oesophagectomy. All other outcomes were comparable, with no significant differences (Tables 2 and 3).

\section{Pathological outcomes}

The incidence of pathological outcomes is summarized in Table 4. Patients undergoing TMIE had a microscopically radical resection rate (R0) of 93.8 per cent compared with 93.2 per cent for hybrid oesophagectomy and 89.2 per cent for open oesophagectomy, with no significant differences between groups.

The median number of resected lymph nodes in TMIE was comparable to that for the other procedures (Table 4). This was also the case when hybrid was compared with open oesophagectomy (standardized coefficient $-1.18, P=0.091$ ). The numbers of positive resected lymph nodes were also comparable between the three surgical techniques.

Tables S1-S3 summarize all postoperative complications registered in the ECCG database.

\section{Discussion}

This study investigated the incidence of postoperative complications after TMIE, hybrid, and open Ivor Lewis oesophagectomy using data from the IESG. Patients undergoing TMIE had a lower pneumonia rate and shorter hospital stay than those treated using hybrid or open approaches. The rate of anastomotic leakage, however, was significantly higher in patients undergoing TMIE.

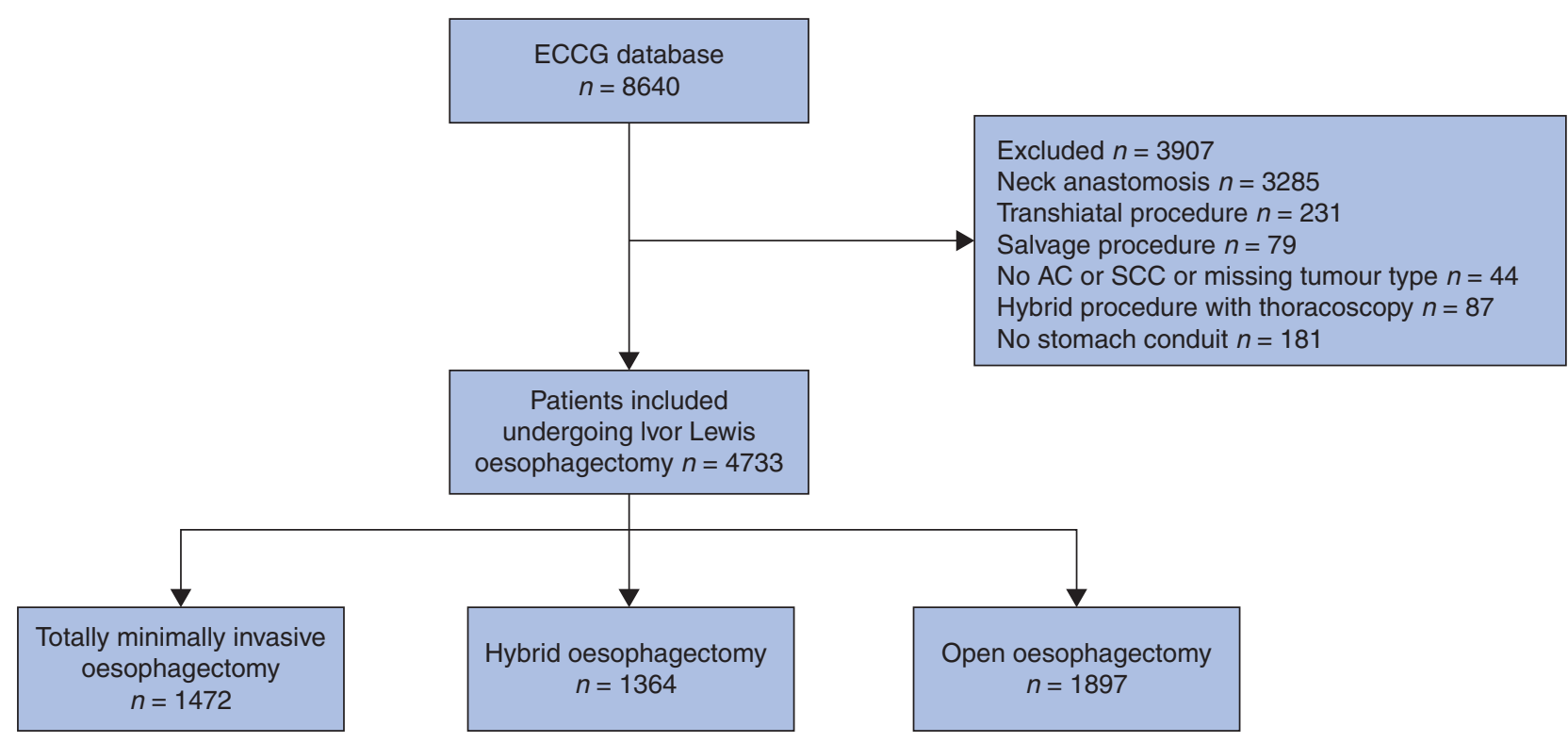

Fig. 1 Study flow diagram showing included patients who underwent Ivor Lewis oesophagectomy 
Table 1 Basic characteristics of patients undergoing totally minimally invasive, hybrid or open Ivor Lewis oesophagectomy

\begin{tabular}{|c|c|c|c|}
\hline & $\begin{array}{c}\text { TMIE } \\
(n=1472)\end{array}$ & $\begin{array}{c}\text { Hybrid }^{\dagger} \\
(n=1364)\end{array}$ & $\begin{array}{c}\text { Open } \\
(n=1897)\end{array}$ \\
\hline Age (years)* & $65(58-71)$ & $64(57-71)$ & $65(58-71)$ \\
\hline Women & $269(18)$ & $241(18)$ & $332(18)$ \\
\hline Co-morbidities present & $920(63)$ & $1183(87)$ & $1468(77)$ \\
\hline \multicolumn{4}{|l|}{ WHO performance status } \\
\hline 0 & $841(57)$ & $617(45)$ & $1029(54)$ \\
\hline 1 & $580(39)$ & $571(42)$ & $798(42)$ \\
\hline 2 & 48 (3.3) & $130(9.5)$ & $60(3)$ \\
\hline 3 & $3(<1)$ & $45(3.3)$ & $10(<1)$ \\
\hline 4 & $0(0)$ & $1(<1)$ & $0(0)$ \\
\hline \multicolumn{4}{|l|}{ ASA fitness grade } \\
\hline I & 145 (9.9) & $118(8.7)$ & $158(8)$ \\
\hline II & $667(45)$ & $758(56)$ & $875(46)$ \\
\hline III & $632(43)$ & $487(36)$ & $860(45)$ \\
\hline IV & $28(1.9)$ & $1(<1)$ & $3(<1)$ \\
\hline $\mathrm{V}$ & $0(0)$ & $0(0)$ & $1(<1)$ \\
\hline \multicolumn{4}{|l|}{ Tumour location } \\
\hline $\begin{array}{l}\text { Proximal } 1 / 2 \text { of } \\
\text { esophagus }\end{array}$ & $41(2.8)$ & $81(5.9)$ & $68(4)$ \\
\hline Distal 1/2 of esophagus & $852(58)$ & $757(56)$ & $863(46)$ \\
\hline GOJ & $558(38)$ & $505(37)$ & $920(49)$ \\
\hline Missing & $21(1.4)$ & $21(1.5)$ & $46(2)$ \\
\hline \multicolumn{4}{|l|}{ Histology } \\
\hline Adenocarcinoma & $895(61)$ & $732(54)$ & $1072(57)$ \\
\hline Squamous cell carcinoma & $146(9.9)$ & $134(9.8)$ & $191(10)$ \\
\hline $\begin{array}{l}\text { Adenosquamous cell } \\
\text { carcinoma }\end{array}$ & $6(<1)$ & $3(<1)$ & $9(<1)$ \\
\hline Missing & $425(29)$ & $495(36)$ & $625(33)$ \\
\hline \multicolumn{4}{|l|}{ Clinical T category $\ddagger$} \\
\hline сTO & $4(<1)$ & $5(<1)$ & $3(<1)$ \\
\hline cTis & $7(<1)$ & $4(<1)$ & $15(<1)$ \\
\hline CT1 & $139(9.4)$ & $91(6.7)$ & $131(7)$ \\
\hline cT2 & $258(18)$ & $166(12)$ & $278(15)$ \\
\hline CT3 & $944(64)$ & $1043(77)$ & $1302(69)$ \\
\hline CT4 & $77(5.2)$ & $26(1.9)$ & $90(5)$ \\
\hline $\mathrm{cTx}$ & $22(1.5)$ & $8(<1)$ & $32(2)$ \\
\hline Missing & $21(1.4)$ & $21(1.5)$ & $46(2)$ \\
\hline \multicolumn{4}{|l|}{ Clinical N category $\ddagger$} \\
\hline cNO & $544(37)$ & $236(17)$ & $728(38)$ \\
\hline $\mathrm{cN} 1$ & $554(38)$ & $403(30)$ & $690(36)$ \\
\hline $\mathrm{cN} 2$ & 226 (15) & $100(7.3)$ & 284 (15) \\
\hline cN3 & $32(2.2)$ & $11(<1)$ & $61(3)$ \\
\hline $\mathrm{cNx}$ & $95(6.5)$ & $593(44)$ & $88(5)$ \\
\hline Missing & $21(1.4)$ & $21(1.5)$ & $46(2)$ \\
\hline \multicolumn{4}{|l|}{ Preoperative treatment } \\
\hline None & 265 (18) & 234 (17) & 354 (19) \\
\hline Chemoradiotherapy & $973(66)$ & $683(50)$ & 765 (40) \\
\hline Chemotherapy & $212(14)$ & $424(31)$ & 731 (39) \\
\hline Radiotherapy & $1(<1)$ & $2(<1)$ & $1(<1)$ \\
\hline Missing & $21(1.4)$ & $21(1.5)$ & $46(2.4)$ \\
\hline
\end{tabular}

Values in parentheses are percentages unless indicated otherwise; *values are median (i.q.r.). ${ }^{\dagger}$ Laparoscopically assisted oesophagectomy (laparoscopy and thoracotomy). ${ }^{\ddagger}$ Clinical Tumor and Nodal stage. TMIE, totally minimally invasive oesophagectomy; GOJ, gastro-oesophageal junction.

No differences were reported between hybrid or open Ivor Lewis approaches.

A recently published meta-analysis ${ }^{27}$ of non-randomized studies compared TMIE with hybrid oesophagectomy. Four studies were pooled that reported on the incidence of pneumonia, including 297 patients in total. The authors did not report a higher incidence of pneumonia for hybrid oesophagectomy compared with TMIE. However, there was heterogeneity in the definitions of pneumonia between the studies. The present study, which used a uniform definition of pneumonia from the ECCG and compared over 2800 patients undergoing TMIE or hybrid Ivor Lewis oesophagectomy, reported a statistically significant difference in
Table 2 Incidence of postoperative complications and duration of hospital stay in patients undergoing totally minimally invasive, hybrid or open Ivor Lewis oesophagectomy

\begin{tabular}{lccc}
\hline & $\begin{array}{c}\text { TMIE } \\
(n=1472)\end{array}$ & $\begin{array}{c}\text { Hybrid } \\
(n=1364)\end{array}$ & $\begin{array}{c}\text { Open } \\
(n=1897)\end{array}$ \\
\hline Pneumonia & $160(10.9)$ & $222(16.3)$ & $331(17.4)$ \\
Anastomotic leakage & $222(15.1)$ & $146(10.7)$ & $139(7.3)$ \\
$\quad$ Type I & $39(2.6)$ & $19(1.4)$ & $51(2.7)$ \\
$\quad$ Type II & $113(7.7)$ & $79(5.8)$ & $51(2.7)$ \\
$\quad$ Type III & $70(4.8)$ & $48(3.5)$ & $37(1.9)$ \\
Complications & & & \\
$\quad$ Any & $881(59.9)$ & $855(62.7)$ & $1100(58.0)$ \\
$\quad$ Major (CD IIIb) & $283(19.2)$ & $219(16.1)$ & $298(15.7)$ \\
Escalation of care & $183(12.4)$ & $198(14.5)$ & $516(27.2)$ \\
Readmission within 30 days & $191(13.0)$ & $81(5.9)$ & $184(9.7)$ \\
Duration of hospital stay (days) & $10(8-16)$ & $14(11-19)$ & $11(9-16)$ \\
90-day mortality & $65(4.4)$ & $46(3.4)$ & $75(4.0)$ \\
& & &
\end{tabular}

Values in parentheses are percentages unless indicated otherwise; *values are median (i.q.r.). TMIE, totally minimally invasive oesophagectomy; $C D$, Clavien-Dindo.

pneumonia rate favouring TMIE over hybrid and open Ivor Lewis oesophagectomy. This higher rate for laparoscopically assisted hybrid and open oesophagectomy most probably reflects the more invasive thoracic procedure as both required a thoracotomy. The present results confirmed the findings of the randomized TIME $^{13}$ and ROBOT ${ }^{14}$ trials, which compared TMIE or robotically-assisted minimally invasive oesophagectomy with open oesophagectomy, and showed a decrease in the rate of pneumonia and pulmonary complications. However, in the present study, there was no difference in pneumonia rate between open and hybrid oesophagectomy, even though the approaches compared were the same as those in the MIRO trial, which randomized and compared patients undergoing either a hybrid (laparoscopy and open thoracotomy) or open oesophagectomy. That trial $^{18}$ reported a significant decrease in major pulmonary complications for hybrid oesophagectomy, defined as pulmonary complications of at least Clavien-Dindo grade II. The pneumonia rates, however, were not analysed separately. In the database used for the present study, the severity of postoperative complications according to the Clavien-Dindo classification was not specified for each complication, making it difficult to make direct comparisons with that study.

In the present study, a higher anastomotic leakage rate was reported for TMIE than hybrid oesophagectomy. The meta-analysis $^{27}$ that compared TMIE with hybrid oesophagectomy reported a significant increase in anastomotic leakage rate for TMIE compared with hybrid Ivor Lewis oesophagectomy. This was most probably due to the technically challenging minimally invasive intrathoracic anastomosis. Hypothetically, the increase in anastomotic leakage rate for TMIE in the present study reflects a proficiency gain curve in centres during implementation of this new technique, as collection of data took place while TMIE was being implemented. If so, it can be expected that anastomotic leakage rates will drop after more patients have been treated ${ }^{17}$. However, after adjustment for interhospital variability, the increased anastomotic leakage rate remained for TMIE. Furthermore, hospitals that undertook most TMIE procedures did not have the lowest anastomotic leakage rates per se, which is partly reflected by the estimated rate of variability which can be explained by interhospital differences. Finally, it is remarkable that, despite a decrease in pneumonia, the anastomotic leakage rate for TMIE was higher even though these complications often coincide. 
Table 3 Multilevel models comparing postoperative complications after totally minimally invasive, hybrid or open Ivor Lewis oesophagectomy

\begin{tabular}{|c|c|c|c|c|c|c|}
\hline & \multicolumn{2}{|c|}{ TMIE versus hybrid $^{\dagger}$} & \multicolumn{2}{|c|}{ TMIE versus open $\dagger$} & \multicolumn{2}{|c|}{ Open versus hybrid $\dagger$} \\
\hline Pneumonia & $0.56(0.40,0.80)$ & 0.001 & $0.60(0.42,0.84)$ & 0.003 & $0.99(0.74,1.32)$ & 0.948 \\
\hline Rate & $1.47(1.01,2.13)$ & 0.045 & $1.73(1.26,2.38)$ & $<0.001$ & $0.79(0.52,1.20)$ & 0.267 \\
\hline Severity (type I-II versus III) & $0.57(0.27,1.18)$ & 0.131 & $0.95(0.46,1.96)$ & 0.886 & $0.61(0.29,1.27)$ & 0.188 \\
\hline \multicolumn{7}{|l|}{ Complications } \\
\hline Escalation of care & $0.89(0.63,1.26)$ & 0.505 & $0.62(0.36,1.07)$ & 0.09 & $1.39(0.81,2.36)$ & 0.229 \\
\hline Readmission within 30 days & $0.97(0.48,1.97)$ & 0.940 & $1.16(0.73,1.86)$ & 0.534 & $0.73(0.39,1.37)$ & 0.329 \\
\hline Duration of hospital stay & $-2.6(-5.0,-0.24)^{*}$ & 0.041 & $-2.2(-3.8,-0.5)^{*}$ & 0.027 & $-0.3(-2.8,2.0)^{*}$ & 0.779 \\
\hline 90-day mortality & $1.01(0.51,2.01)$ & 0.978 & $0.83(0.47,1.44)$ & 0.497 & $1.65(0.80,3.40)$ & 0.179 \\
\hline
\end{tabular}

Values in parentheses are 95 per cent confidence intervals; "Standardized coefficients. ${ }^{\dagger}$ Reference group. TMIE, totally minimally invasive oesophagectomy; OR, odds ratio; CD, Clavien-Dindo. Analyses were adjusted for random hospital effects, tumour histology, preoperative treatment, age, sex, WHO performance stats, cT category, $\mathrm{cN}$ category, and tumour location.

Table 4 Pathological outcomes after totally minimally invasive, hybrid or open Ivor Lewis oesophagectomy

\begin{tabular}{|c|c|c|c|}
\hline & $\begin{array}{c}\text { TMIE } \\
(n=1472)\end{array}$ & $\begin{array}{c}\text { Hybrid } \\
(n=1364)\end{array}$ & $\begin{array}{c}\text { Open } \\
(n=1897)\end{array}$ \\
\hline \multicolumn{4}{|l|}{ Pathological T category } \\
\hline pTO & $280(19.0)$ & $239(17.5)$ & $253(13.3)$ \\
\hline pTis & $11(0.7)$ & $2(0.1)$ & $9(0.5)$ \\
\hline pT1 & $324(22.0)$ & $282(20.7)$ & 345 (18.2) \\
\hline pT2 & $227(15.4)$ & $197(14.4)$ & $269(14.2)$ \\
\hline pT3 & $569(38.7)$ & $577(42.3)$ & $875(46.1)$ \\
\hline pT4 & $33(2.2)$ & $36(2.6)$ & $91(4.8)$ \\
\hline $\mathrm{pTx}$ & $7(0.5)$ & $2(0.1)$ & $9(0.5)$ \\
\hline Missing & $21(1.4)$ & $21(1.5)$ & $46(2.4)$ \\
\hline \multicolumn{4}{|l|}{ Pathological N category } \\
\hline pNO & $855(58.1)$ & $743(54.5)$ & $953(50.2)$ \\
\hline pN1 & $310(21.1)$ & $305(22.4)$ & $449(23.7)$ \\
\hline pN2 & $187(12.7)$ & $165(12.1)$ & $253(13.3)$ \\
\hline pN3 & $98(6.7)$ & $128(9.4)$ & $195(10.3)$ \\
\hline $\mathrm{pNx}$ & $1(0.1)$ & $2(0.1)$ & $1(0.1)$ \\
\hline Missing & $21(1.4)$ & $21(1.5)$ & $46(2.4)$ \\
\hline \multicolumn{4}{|l|}{ Pathological M category } \\
\hline pMo & $1363(92.6)$ & $1254(91.9)$ & $1592(83.9)$ \\
\hline pM1 & $13(0.9)$ & $25(1.8)$ & $46(2.4)$ \\
\hline $\mathrm{pMx}$ & $75(5.1)$ & $64(4.7)$ & $213(11.2)$ \\
\hline Missing & $21(1.4)$ & $21(1.5)$ & $46(2.4)$ \\
\hline \multicolumn{4}{|l|}{ Radicality of resection } \\
\hline R0 & $1381(93.8)$ & $1271(93.2)$ & 1693 (89.2) \\
\hline R1 & $70(4.8)$ & $70(5.1)$ & $152(8.0)$ \\
\hline R2 & $0(0)$ & $2(0.1)$ & $6(0.3)$ \\
\hline Missing & $21(1.4)$ & $21(1.5)$ & $46(2.4)$ \\
\hline No. of resected lymph nodes* & $30(21-40)$ & $29(22-37)$ & $26(19-34)$ \\
\hline $\begin{array}{l}\text { No. of lymph nodes } \\
\text { containing tumour cells* }\end{array}$ & $0(0-2)$ & $0(0-2)$ & $0(0-2)$ \\
\hline
\end{tabular}

Values in parentheses are percentages unless indicated otherwise; *values are median (i.q.r.). TMIE, totally minimally invasive oesophagectomy.

A previous meta-analysis ${ }^{8}$ reported a decrease in overall survival after development of anastomotic leakage or pneumonia in patients undergoing any type of oesophagectomy. Furthermore, in a study ${ }^{10}$ that included only patients undergoing TMIE, anastomotic leakage resulted in decreased long-term survival compared with that among patients who did not develop anastomotic leakage. In the present study, the 90-day postoperative mortality rate was, however, comparable between the groups. The only way to definitively assess the importance of differences in postoperative complications is to prospectively and directly compare both surgical techniques, powered on outcomes such as overall survival and long-term postoperative health-related quality of life.

To the authors' knowledge, this is the largest study comparing postoperative outcomes after different approaches to Ivor Lewis oesophagectomy for oesophageal cancer. The IESG ${ }^{19,20}$ previously reached an international consensus on standardized reporting of the most important postoperative complications. This resulted in a robust and standardized comparison between surgical approaches used throughout the world.

The present study has some limitations. Healthcare personnel were not blinded to the procedure performed. The duration of hospital stay could have been influenced by surgical approach as known to the healthcare provider in the surgical ward. Some variables that would be of value in the present study were not reported in the database. The anastomotic technique used (end to side, side to side, circular or linear stapled) in oesophagectomy has been associated with the risk of anastomotic leakage ${ }^{28}$. These anastomotic techniques were not registered in the database and could not therefore be adjusted for. Other variables of interest but not reported in the database are the rate of surgical conversion and survival after different surgical techniques. Although adjustment was made for interhospital differences, international outcomes for oesophagectomy remain variable and can still lead to variability in comparison ${ }^{29,30}$. Finally, although the definition of pneumonia was highly standardized, there was no clear definition developed specifically for postoperative pneumonia. A standardized severity score should be reported for pneumonia to gain insight into its possible impact in the postoperative setting. To the best of the authors' knowledge, the only randomized trial including both laparoscopically assisted hybrid oesophagectomy and TMIE (both McKeown and Ivor Lewis) is the ROMIO trial ${ }^{31}$. This ongoing trial, however, is randomizing only a small number of patients to the TMIE group by means of a substudy with the aim of evaluating the safety of TMIE. Overall survival and postoperative complications will therefore probably not be evaluated with sufficient power to find an improved outcome for either surgical technique.

This study has shown no clear advantage for either TMIE, hybrid or open Ivor Lewis oesophagectomy when performed in daily clinical practice, and the choice of surgical approach should depend on centre experience, centre volume, and surgeon preferences. Minimally invasive techniques should be further developed 
to minimize postoperative complications, such as anastomotic leakage.

\section{Collaborators}

J. Jan B. van Lanschot (Erasmus MC Cancer Institute, Rotterdam, the Netherlands); Egle Jezerskyte (Amsterdam University Medical Centre, University of Amsterdam, Amsterdam Cancer Centre, Amsterdam, the Netherlands); Wietse J. Eshuis (Amsterdam University Medical Centre, University of Amsterdam, Amsterdam Cancer Centre, Amsterdam, the Netherlands); Jelle Ruurda (University Medical Centre Utrecht, Utrecht, the Netherlands); Sylvia van der Horst (University Medical Centre Utrecht, Utrecht, the Netherlands); Arjen van der Veen (University Medical Centre Utrecht, Utrecht, the Netherlands); Gino Kuiper(University Medical Centre Utrecht, Utrecht, the Netherlands); Toni Lerut (University Hospitals Leuven, Leuven Cancer Institute, Leuven, Belgium); Johnny Moons(University Hospitals Leuven, Leuven Cancer Institute, Leuven, Belgium); Marc Bludau (University Hospital of Cologne, Cologne, Germany); Christiane J. Bruns (University Hospital of Cologne, Cologne, Germany); Fredrik Klevebro (Intervention and Technology, Karolinska Institutet and Department of Upper Abdominal Diseases, Karolinska University Hospital, Stockholm, Sweden); Masaru Hayami (Intervention and Technology, Karolinska Institutet and Department of Upper Abdominal Diseases, Karolinska University Hospital, Stockholm, Sweden); Xavier B. D'Journo (Aix-Marseille University, North Hospital, Marseille, France); Delphine Trousse (Aix-Marseille University, North Hospital, Marseille, France); Geoffrey Brioude (Aix-Marseille University, North Hospital, Marseille, France); Richard Hardwick (Cambridge Oesophago-Gastric Centre, Addenbrookes Hospital, Cambridge, UK); Peter Safranek (Cambridge Oesophago-Gastric Centre, Addenbrookes Hospital, Cambridge, UK); John Bennett (Cambridge Oesophago-Gastric Centre, Addenbrookes Hospital, Cambridge, UK); Andrew Hindmarsh (Cambridge Oesophago-Gastric Centre, Addenbrookes Hospital, Cambridge, UK); Vijay Sujendran (Cambridge Oesophago-Gastric Centre, Addenbrookes Hospital, Cambridge, UK); John R. O'Neill (Cambridge Oesophago-Gastric Centre, Addenbrookes Hospital, Cambridge, UK); Arnulf H. Hoelscher (Centre for Esophageal Diseases, Elisabeth Hospital Essen, University Medicine Essen, Essen, Germany); Martin Hemmerich (Centre for Esophageal Diseases, Elisabeth Hospital Essen, University Medicine Essen, Essen, Germany); Guillaume Piessen (Claude Huriez University Hospital, Lille, France); Marguerite Messier (Claude Huriez University Hospital, Lille, France); Sebastien Degisors (Claude Huriez University Hospital, Lille, France); Frederiek Nuytens (Claude Huriez University Hospital, Lille, France); Blair A. Jobe (Esophageal and Lung Institute, Allegheny Health Network, Pittsburgh, PA, USA); Ali H. Zaidi (Esophageal and Lung Institute, Allegheny Health Network, Pittsburgh, PA, USA); Andrew Davies (Guy's \& St Thomas' NHS Foundation Trust, London, UK); Janine Zylstra (Guy's \& St Thomas' NHS Foundation Trust, London, UK); James Gossage (Guy's \& St Thomas' NHS Foundation Trust, London, UK); Cara Baker (Guy's \& St Thomas' NHS Foundation Trust, London, UK); Mark Kelly (Guy's \& St Thomas' NHS Foundation Trust, London, UK); Paul M. Schneider (Hirslanden Medical Centre, Zurich, Switzerland); Simone Schillinger (Hirslanden Medical Centre, Zurich, Switzerland); (Hirslanden Medical Centre, Zurich, Switzerland); Manuel Pera (Hospital Universitario del Mar, Barcelona, Spain); Marta Gimeno
(Hospital Universitario del Mar, Barcelona, Spain); Yuko Kitagawa (Keio University, Tokyo, Japan); Hirofumi Kawakubo (Keio University, Tokyo, Japan); Satoru Matsuda (Keio University, Tokyo, Japan); Yuki Hirata (Keio University, Tokyo, Japan); Christopher R. Morse (Massachusetts General Hospital, Boston, MA, USA); Julie Garrity (Massachusetts General Hospital, Boston, MA, USA); Wayne L. Hofstetter (MD Anderson Cancer Centre, Houston, TX, USA); Huawei Tang (MD Anderson Cancer Centre, Houston, TX, USA); Daniela Molena (Memorial Sloan Kettering Cancer Centre, New York City, NY, USA); Manjit Bains (Memorial Sloan Kettering Cancer Centre, New York City, NY, USA); Joe Dycoco (Memorial Sloan Kettering Cancer Centre, New York City, NY, USA); Kristen Busalacchi (Memorial Sloan Kettering Cancer Centre, New York City, NY, USA); Rebecca Carr (Memorial Sloan Kettering Cancer Centre, New York City, NY, USA); David R. Jones (Memorial Sloan Kettering Cancer Centre, New York City, NY, USA); Jimmy B.Y. So (National University Hospital, Singapore, Singapore); Asim Shabbir (National University Hospital, Singapore, Singapore); Arul Immanuel (Northern Oesophagogastric Cancer Unit, Royal Victoria Infirmary, Newcastle upon Tyne, UK); Michael Griffin (Northern Oesophagogastric Cancer Unit, Royal Victoria Infirmary, Newcastle upon Tyne, UK); Helen Jaretzke (Northern Oesophagogastric Cancer Unit, Royal Victoria Infirmary, Newcastle upon Tyne, UK); Simon L. Parsons (Nottingham University Hospitals NHS Trust, Nottingham, UK); Neil T. Welch (Nottingham University Hospitals NHS Trust, Nottingham, UK); Ravinder Vohra (Nottingham University Hospitals NHS Trust, Nottingham, UK); James A. Catton (Nottingham University Hospitals NHS Trust, Nottingham, UK); John H. Saunders (Nottingham University Hospitals NHS Trust, Nottingham, UK); Fady Yanni (Nottingham University Hospitals NHS Trust, Nottingham, UK); Daniela Zanotti (Nottingham University Hospitals NHS Trust, Nottingham, UK); Pritam Singh (Nottingham University Hospitals NHS Trust, Nottingham, UK); Michael H. Larsen (Odense University Hospital, Odense, Denmark); Larsen Nicolaj (Odense University Hospital, Odense, Denmark); Marcus Stilling (Odense University Hospital, Odense, Denmark); James P. Dolan (Oregon Health and Science University, Portland, OR, USA); Stephanie G. Wood (Oregon Health and Science University, Portland, OR, USA); Charlie C. Borzy (Oregon Health and Science University, Portland, OR, USA); Kayla Siemens (Oregon Health and Science University, Portland, OR, USA); Nick Maynard (Oxford University Hospitals NHS Foundation Trust, Oxford, UK); John Findlay (Oxford University Hospitals NHS Foundation Trust, Oxford, UK); Stephen Ash (Oxford University Hospitals NHS Foundation Trust, Oxford, UK); Mark Smithers (Princess Alexandra Hospital, University of Queensland, Brisbane, Australia); Iain Thomson (Princess Alexandra Hospital, University of Queensland, Brisbane, Australia); Andrew Barbour (Princess Alexandra Hospital, University of Queensland, Brisbane, Australia); Janine Thomas (Princess Alexandra Hospital, University of Queensland, Brisbane, Australia); Sonia Puig (Queen Elizabeth Hospital Birmingham, UHB Foundation Trust, Birmingham, UK); John Whiting (Queen Elizabeth Hospital Birmingham, UHB Foundation Trust, Birmingham, UK); Simon Y.K. Law (Queen Mary Hospital, The University of Hong Kong, Hong Kong SAR, China); Ian Y. Wong (Queen Mary Hospital, The University of Hong Kong, Hong Kong SAR, China); Jeannette Kwok (Queen Mary Hospital, The University of Hong Kong, Hong Kong SAR, China); Andrew Kennedy (Royal Victoria Hospital, Belfast, Northern Ireland); Raymond Kennedy, (Royal Victoria Hospital, Belfast, Northern Ireland); Wang K. Ning (Sichuan Cancer Hospital \& Institute, Chengdu, China); 
Fang Qiang (Sichuan Cancer Hospital \& Institute, Chengdu, China); Han YongTao (Sichuan Cancer Hospital \& Institute, Chengdu, China); Peng Lin (Sichuan Cancer Hospital \& Institute, Chengdu, China); Xiao WenGuang (Sichuan Cancer Hospital \& Institute, Chengdu, China); John V. Reynolds (St. James's Hospital Trinity College, Dublin, Ireland); Sinead King (St. James's Hospital Trinity College, Dublin, Ireland); Ravi Narayanasamy (St. James's Hospital Trinity College, Dublin, Ireland); C.S. Pramesh (Tata Memorial Centre, Mumbai, India); Apurva Ashok (Tata Memorial Centre, Mumbai, India); Mark K. Ferguson (The University of Chicago Medicine, Chicago, IL, USA); Amy Durkin-Celauro (The University of Chicago Medicine, Chicago, IL, USA); Catherine Staub (The University of Chicago Medicine, Chicago, IL, USA); Gail E. Darling (Toronto General Hospital, Toronto, Canada); Emma Small (Toronto General Hospital, Toronto, Canada); Tim Underwood (University Hospital Southampton NHS Foundation Trust, Southampton, UK); James Byme (University Hospital Southampton NHS Foundation Trust, Southampton, UK); Jamie Kelly (University Hospital Southampton NHS Foundation Trust, Southampton, UK); Fergus Noble (University Hospital Southampton NHS Foundation Trust, Southampton, UK); Donna Sharland (University Hospital Southampton NHS Foundation Trust, Southampton, UK); Rachel Fraser (University Hospital Southampton NHS Foundation Trust, Southampton, UK); Rob Walker (University Hospital Southampton NHS Foundation Trust, Southampton, UK); Saqib Rahman (University Hospital Southampton NHS Foundation Trust, Southampton, UK); Ben Grace, (University Hospital Southampton NHS Foundation Trust, Southampton, UK); Andrew C. Chang, (University of Michigan Health System, Ann Arbor, MI, USA); Judy Miller, (University of Michigan Health System, Ann Arbor, MI, USA); Shari Barnett, (University of Michigan Health System, Ann Arbor, MI, USA); Ivan Cecconello, (University of Sao Paulo School of Medicine, Sao Paulo, Brazil); Ulysses Ribeiro Jr, (University of Sao Paulo School of Medicine, Sao Paulo, Brazil); Rubens A. A. Sallum, (University of Sao Paulo School of Medicine, Sao Paulo, Brazil); Giovanni de Manzoni, (University of Verona, Verona, Italy); Jacopo Weindelmayer, (University of Verona, Verona, Italy); Carlo Alberto De Pasqual, (University of Verona, Verona, Italy); Riccardo Rosati, (San Raffaele University, Milan, Italy); Paolo Parise, (San Raffaele University, Milan, Italy); Andrea Cossu, (San Raffaele University, Milan, Italy); Francesco Puccetti, Simonetta Massaron, (San Raffaele University, Milan, Italy); Vita-Salute (San Raffaele University, Milan, Italy); Madhan K. Kuppusamy, (Virginia Mason Medical Centre, Seattle, WA, USA); Bonnie Marston, (Virginia Mason Medical Centre, Seattle, WA, USA); Donald E. Low, (Virginia Mason Medical Centre, Seattle, WA, USA).

\section{Acknowledgements}

B.J.v.d.W. and E.R.C.H. are joint first authors, and S.M.L. and M.I.v.B.H. are joint senior authors of this article.The authors acknowledge D. Nieboer, Department of Public Health, Erasmus-University Medical Centre, for statistical assistance during the design of the study.

Disclosure. The authors declare no conflict of interest.

\section{Supplementary material}

Supplementary material is available at BJS online.

\section{References}

1. Shapiro J, van Lanschot JJB, Hulshof MCCM, van Hagen P, van Berge Henegouwen MI, Wijnhoven BPL et al.; CROSS Study Group. Neoadjuvant chemoradiotherapy plus surgery versus surgery alone for oesophageal or junctional cancer (CROSS): long-term results of a randomised controlled trial. Lancet Oncol 2015;16:1090-1098.

2. van Hagen P, Hulshof MCCM, van Lanschot JJB, Steyerberg EW, van Berge Henegouwen MI, Wijnhoven BPL et al. Preoperative chemoradiotherapy for esophageal or junctional cancer. N Engl J Med 2012;366:2074-2084.

3. Yang H, Liu H, Chen Y, Zhu C, Fang W, Yu Z et al.; AME Thoracic Surgery Collaborative Group. Neoadjuvant chemoradiotherapy followed by surgery versus surgery alone for locally advanced squamous cell carcinoma of the esophagus (NEOCRTEC5010): a phase III multicenter, randomized, open-label clinical trial. J Clin Oncol 2018;36:2796-2803.

4. Shah MA, Kennedy EB, Catenacci DV, Deighton DC, Goodman KA, Malhotra NK et al. Treatment of locally advanced esophageal carcinoma: ASCO guideline. J Clin Oncol 2020;38:2677-2694.

5. Viklund P, Lindblad M, Lu M, Ye W, Johansson J, Lagergren J. Risk factors for complications after esophageal cancer resection: a prospective population-based study in Sweden. Ann Surg 2006;243:204-211.

6. Noordman BJ, Verdam MGE, Lagarde SM, Shapiro J, Hulshof MCCM, van Berge Henegouwen MI et al.; CROSS Study Group. Impact of neoadjuvant chemoradiotherapy on health-related quality of life in long-term survivors of esophageal or junctional cancer: results from the randomized CROSS trial. Ann Oncol 2018;29:445-451.

7. Markar SR, Zaninotto G, Castoro C, Johar A, Lagergren P, Elliott JA, et al. Lasting symptoms after esophageal resection (LASER): European multicenter cross-sectional study. Ann Surg 2020.

8. Booka E, Takeuchi H, Suda K, Fukuda K, Nakamura R, Wada N et al. Meta-analysis of the impact of postoperative complications on survival after oesophagectomy for cancer. BJS Open 2018;2: 276-284

9. Linden PA, Towe CW, Watson TJ, Low DE, Cassivi SD, GrauSepulveda M et al. Mortality after esophagectomy: analysis of individual complications and their association with mortality. J Gastrointest Surg 2020;24:1948-1954.

10. Fransen LFC, Berkelmans GHK, Asti E, Henegouwen MIVB, Berlth F, Bonavina L, et al. The effect of postoperative complications after minimally invasive esophagectomy on long-term survival: an International Multicenter Cohort Study. Ann Surg 2020.

11. Lagarde SM, de Boer JD, ten Kate FJ, Busch OR, Obertop H, van Lanschot JJ. Postoperative complications after esophagectomy for adenocarcinoma of the esophagus are related to timing of death due to recurrence. Ann Surg 2008;247:71-76.

12. Gisbertz SS, Hagens ERC, Ruurda JP, Schneider PM, Tan LJ, Domrachev SA et al. The evolution of surgical approach for esophageal cancer. Ann N Y Acad Sci 2018;1434:149-155.

13. Biere SSAY, van Berge Henegouwen MI, Maas KW, Bonavina L, Rosman C, Garcia JR et al. Minimally invasive versus open oesophagectomy for patients with oesophageal cancer: a multicentre, open-label, randomised controlled trial. Lancet 2012;379: 1887-1892.

14. van der Sluis PC, van der Horst S, May AM, Schippers C, Brosens LAA, Joore HCA et al. Robot-assisted minimally invasive thoracolaparoscopic esophagectomy versus open transthoracic 
esophagectomy for resectable esophageal cancer: a randomized controlled trial. Ann Surg 2019;269:621-630.

15. Markar SR, Ni M, Gisbertz SS, van der Werf L, Straatman J, van der Peet D et al.; Dutch Upper GI Cancer Audit and TIME Study Group. Implementation of minimally invasive esophagectomy from a randomized controlled trial setting to national practice. J Clin Oncol 2020;38:2130-2139.

16. Seesing MFJ, Gisbertz SS, Goense L, van Hillegersberg R, Kroon HM, Lagarde SM et al. A propensity score matched analysis of open versus minimally invasive transthoracic esophagectomy in the Netherlands. Ann Surg 2017;266:839-846.

17. van Workum F, Stenstra MHBC, Berkelmans GHK, Slaman AE, van Berge Henegouwen MI, Gisbertz SS et al. Learning curve and associated morbidity of minimally invasive esophagectomy: a retrospective multicenter study. Ann Surg 2019;269:88-94.

18. Mariette C, Markar SR, Dabakuyo-Yonli TS, Meunier B, Pezet D, Collet D et al. Hybrid minimally invasive esophagectomy for esophageal cancer. N Engl J Med 2019;380:152-162.

19. Low DE, Alderson D, Cecconello I, Chang AC, Darling GE, D'journo XB et al. International consensus on standardization of data collection for complications associated with esophagectomy: Esophagectomy Complications Consensus Group (ECCG). Ann Surg 2015;262:286-294.

20. Low DE, Kuppusamy MK, Alderson D, Cecconello I, Chang AC, Darling $G$ et al. Benchmarking complications associated with esophagectomy. Ann Surg 2019;269:291-298.

21. von Elm E, Altman DG, Egger M, Pocock SJ, Gøtzsche PC, Vandenbroucke JP et al.; STROBE Initiative. Strengthening the Reporting of Observational Studies in Epidemiology (STROBE) statement: guidelines for reporting observational studies. BMJ 2007;335:806-808.

22. Cunha AB. Nosocomial and Healthcare-Associated Pneumonia. http://emedicine.medscape.com/article/234753-overview (accessed 21 December 2014).

23. American Thoracic Society; Infectious Diseases Society of America. Guidelines for the management of adults with hospital-acquired, ventilator-associated, and healthcare- associated pneumonia. Am J Respir Crit Care Med 2005;171: 388-416.

24. Clavien PA, Barkun J, de Oliveira ML, Vauthey JN, Dindo D, Schulick RD et al. The Clavien-Dindo classification of surgical complications: five-year experience. Ann Surg 2009;250:187-196.

25. Leyland AH, Groenewegen PP. Apportioning variation in multilevel models. In: Multilevel Modelling for Public Health and Health Services Research: Health in Context. Cham: Springer International Publishing, 2020, 89-104.

26. Roderick JAL, Donald BR. Statistical Analysis with Missing Data. Hoboken, NJ, USA: John Wiley \& Sons, 1986.

27. van Workum F, Klarenbeek BR, Baranov N, Rovers MM, Rosman C. Totally minimally invasive esophagectomy versus hybrid minimally invasive esophagectomy: systematic review and meta-analysis. Dis Esophagus 2020;33.

28. Schröder W, Raptis DA, Schmidt HM, Gisbertz SS, Moons J, Asti E et al. Anastomotic techniques and associated morbidity in total minimally invasive transthoracic esophagectomy: results from the EsoBenchmark Database. Ann Surg 2019;270:820-826.

29. Oesophago-Gastric Anastomotic Audit Collaborative: Writing Committee, Steering Committee, National Leads, Site Leads, Collaborators. Mortality from esophagectomy for esophageal cancer across low, middle, and high-income countries: an international cohort study. EurJ Surg Oncol 2021;47:1481-1488.

30. Knight SR, Shaw CA, Pius R, Drake TM, Norman L, Ademuyiwa AO et al.; National Institute for Health Research Global Health Research Unit on Global Surgery. Global variation in postoperative mortality and complications after cancer surgery: a multicentre, prospective cohort study in 82 countries. Lancet 2021; 397:387-397.

31. Brierley RC, Gaunt D, Metcalfe C, Blazeby JM, Blencowe NS, Jepson $\mathrm{M}$ et al. Laparoscopically assisted versus open oesophagectomy for patients with oesophageal cancer-the Randomised Oesophagectomy: Minimally Invasive or Open (ROMIO) study: protocol for a randomised controlled trial (RCT). BMJ Open 2019;9:e030907. 